\title{
NONPARAMETRIC BAYES ESTIMATION OF THE RELIABILITY FUNCTION OF A COHERENT SYSTEM
}

\author{
AKM FAZLUR RAHMAN ${ }^{\star}$ \\ Department of Biostatistics, School of Public Health \\ The University of Alabama at Birmingham, Birmingham, AL 35294, USA \\ Email:frahman@uab.edu \\ EDSEL A. PEÑA \\ Department of Statistics, University of South Carolina, Columbia, SC 29208, USA \\ Email: pena@stat.sc.edu
}

\section{SUMMARY}

Complex coherent systems are the engines driving forward our technological world. A coherent system is composed of components, which could be modules or sub-systems, that interact with each other according to some structure function. For purposes of maintenance and safety considerations, it is of critical importance to gain knowledge of the distribution of the system lifetime, with this distribution being a function of the distributions of the components lifetimes. Since the monitoring of a system ceases upon system failure, at system failure some components will be failed, while others, depending on the structure function, will still be functioning with their lifetimes right-censored by the system lifetime. This paper deals with the estimation of the system lifetime distribution. The inferential framework is nonparametric Bayesian, with partition-based Dirichlet processes (PBDP) assigned as priors on the components lifetime distributions. PBDP are more general than the usual Dirichlet process (DP) priors and are particularly suited as priors in settings with censored data. The resulting estimator of the system life distribution, which is a function of the nonparametric Bayes estimators of the components lifetime distributions, is compared in terms of bias and variance with a product-limit type estimator proposed by Doss, et. al. (Ann. Statist., 1989), which can be obtained as a limit of the proposed estimator. These comparisons, which are facilitated through computer simulations, demonstrate that the proposed estimator possesses some robustness. The proposed estimator is illustrated using a synthetic data for a parallel system with five components.

Keywords and phrases: Nonparametric Bayes methods; Partition based Dirichlet process; Nonparametric prior; PL-type estimator.

AMS Classification: Primary: 62N01; Secondary: 62F15.

\footnotetext{
* Corresponding author

(C) Institute of Statistical Research and Training (ISRT), University of Dhaka, Dhaka 1000, Bangladesh.
} 


\section{Introduction}

Systems abound in nature and in our technological society. Systems are those that are composed of inter-connected components or subsystems to form a unified unit. Computers and computer networks, telephones and telephone infrastructures, cars, roads and highways, homes, trains, airplanes, hospitals, medical equipments, human body, tanks, missiles, military command infrastructures, etc. are examples of systems in engineering, public health, biomedical, and military settings. They could also arise in the economic, political, and sociological sciences, such as a "binary" or 2-state system, the US system of government, US Congress, the US financial and banking infrastructure, the United Nations, economic and military treaties, etc. Coherent systems are those where every component or subsystem is relevant and the improvement of a component or a subsystem could not make the system worse. The reliability of a system over a fixed period of time is the probability that the system functions over this time period. The reliability of a system is a function of the reliabilities of each of its components or subsystems. Naturally we desire systems with high reliability since human lives and the welfare of our society are usually at stake and fully dependent on the proper functioning of the multitude of systems governing our day-to-day living. Thus, as a consequence, it is of prime importance that the reliability of systems could be properly assessed before their actual deployment. This paper is for the purpose of proposing methods for performing statistical inference regarding the reliability of coherent systems which could be useful in the processes of improving the systems.

We briefly review relevant and related published works and then provide a more formal mathematical background by introducing some notation and definitions regarding coherent systems. Moeschberger and David (1971) considered estimation of system lifetime distribution, $F$, under independent and identically distributed (IID) assumptions on the components and in a competing

risks framework. Estimation of $F$ under rank set sampling for $k$-out-of- $K$ systems was considered by Kvam and Samaniego (1994) and Stokes and Sager (1988). Estimation of load sharing properties in a dynamic reliability system was tackled in Kvam and Peña (2005). Joint estimation of components and system reliabilities was addressed in Doss et al. (1989). In addition, inferential problems to assess the risk and reliability of systems has, among others, been considered by Barlow and Hunter (1960), Barlow and Marshall (1967), Barlow and Proschan (1969, 1986), Barlow (1984. 1985, 1986), Boyles et al. (1985), El-Neweihi et al. (1978), Esary and Proschan (1963), Esary et al. (1971, 1970), Hollander and Proschan (1984), Hollander and Peña (1995, 1996ab, 2004), Langberg et al. (1981), Meilijson (1981), Peña and Hollander (2004), Navarro and Rychlik (2007), Polpo and Pereira (2009), Polpo et al. (2013), Walker and Muliere (1997), and Susarla and Van Ryzin (1976). Most of these works employed a frequentist parametric or nonparametric statistical inference framework for the system reliability. This frequentist framework is perfectly acceptable when the postulated parametric assumptions are valid, but it could perform poorly under misspecified models.

In contrast to classical nonparametric framework, the nonparametric Bayesian framework proposed in this paper enables us to incorporate prior knowledge thereby leading to more robust estimation procedures of system reliability. Polpo and Pereira (2009) considered Bayesian estimation of reliability in parallel systems and Polpo et al. (2013) considered Bayesian estimation of reliabilities in a class of coherent systems. In their work, Polpo et al. (2013) assumed that failure times of all the 
systems are known and the components that produced the failure. However, system lifetime could also be right-censored in an experimental setting due to finite monitoring period or system withdrawn from the observation, for example, due to accident. In our work we assumed that systems lifetimes as well as component lifetimes could possibly be right-censored data and not limited to complete observations as considered by Polpo et al. (2013). In our nonparametric Bayesian framework, we assign a partition-based Dirichlet measure (PBDM) prior as introduced in Sethuraman and Hollander (2009) on $F$, or on the component reliability functions. The resulting posterior measures, given the observable data from the coherent systems, are also partition-based Dirichlet measures. The resulting estimator based on a quadratic loss function of the system reliability function $F$ is in closed form being a linear combination of the prior estimate of $F$ and the corresponding nonparametric PL-type estimator of $F$.

We now briefly outline the contents of this paper. Section 2 presents some notation and definitions pertaining to coherent systems and the observable data from coherent systems. In Section 3 we provide background information and results about the PBDM prior and develop the estimators of the system reliability function based on the system data. In Section 4 we obtain the nonparametric Bayes estimators of the components reliability functions and also an estimator of the system reliability function based on component data. We also explore the relationship between our proposed estimator and the corresponding PL-type estimator. Section 5 includes simulation studies and provides comparisons between the proposed estimators and the PL-type estimators in terms of bias and RMSE function, which we note are frequentist framework criteria for assessing the goodness of estimators. Section 6 provides an illustration of the proposed estimators using a synthetic data set and Section 7 provides some concluding remarks, while Section $\mathrm{A}$ is an appendix wherein we gather detail proof of the Theorem 3 .

\section{Mathematical Preliminaries}

We provide our notation and recall some definitions relevant to coherent systems; see Barlow and Proschan (1981) for a comprehensive discussion of coherent systems. A reliability system is composed of a finite number of components, with each component possibly being a subsystem itself or a two-state binary system. For a reliability system with $K$ components, denote the state vector of components by $\mathbf{x}=\left(x_{1}, x_{2}, \ldots, x_{K}\right)$, with $x_{j} \in\{0,1\}$ and such that $x_{j}=1(0)$ means that component $j$ is functioning (failed). The structure function of a reliability system is defined by $\phi:\{0,1\}^{K} \rightarrow\{0,1\}$ such that $\phi(\mathbf{x})=1(0)$ indicates that the system is in a functioning (failed) state. A reliability system is said to be coherent if the structure function $\phi(\mathbf{x})$ satisfies the two conditions: (i) it is nondecreasing in each argument, i.e., a change of state of one and only one component from a failed state to a working state should not cause the system to change from a working state to a failed state; (ii) each component is relevant in the sense that, for each $j \in\{1,2, \ldots, K\}$, there exists an $\mathbf{x} \in\{0,1\}^{K}$ such that $0=\phi\left(\mathbf{x}, 0_{j}\right)<\phi\left(\mathbf{x}, 1_{j}\right)=1$, where $\left(\mathbf{x}, 0_{j}\right)=\left(x_{1}, \ldots, x_{j-1}, 0, x_{j+1}, \ldots, x_{K}\right)$ and $\left(\mathbf{x}, 1_{j}\right)=\left(x_{1}, \ldots, x_{j-1}, 1, x_{j+1}, \ldots, x_{K}\right)$. Hereon, coherent reliability systems will be referred to as systems for brevity.

A series system and a parallel system are two examples of coherent reliability systems with 
respective structure functions

$$
\begin{aligned}
& \phi_{\text {ser }}(\mathbf{x})=\min \left\{x_{1}, x_{2}, \ldots, x_{K}\right\}=\prod_{j=1}^{K} x_{j} \\
& \phi_{\text {par }}(\mathbf{x})=\max \left\{x_{1}, x_{2}, \ldots, x_{K}\right\}=1-\prod_{j=1}^{K}\left(1-x_{j}\right) .
\end{aligned}
$$

The coherent structure function of a $k$-out-of- $K$ system is

$$
\phi_{k: K}(\mathbf{x})=I\left\{\sum_{j=1}^{K} x_{j} \geq k\right\}
$$

with $I(A)=1(0)$ depending on whether event $A$ does (does not) hold. Clearly, a series system ( $K$-out-of- $K$ system) and a parallel system (1-out-of- $K$ ) are the two extreme cases of $k$-out-of$K$ systems, and in fact of all $K$-component systems. Another coherent system is a 3-component series-parallel system with block diagram shown in Figure 1 and whose structure function is

$$
\phi_{s p}\left(x_{1}, x_{2}, x_{3}\right)=\min \left\{x_{1}, \max \left\{x_{2}, x_{3}\right\}\right\}=x_{1} \wedge\left(x_{2} \vee x_{3}\right) .
$$

This series-parallel system functions so long as component 1 and at least one of components 2 or 3 are functioning.

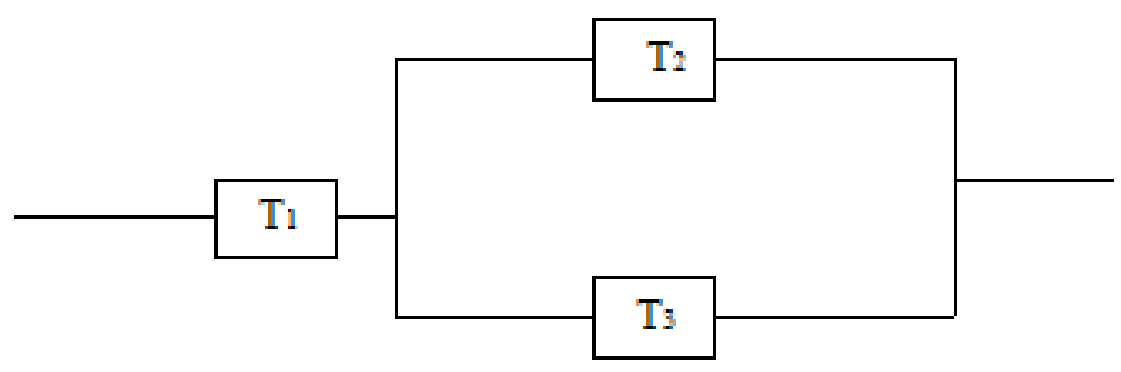

Figure 1: Three Component Series-Parallel System

Let $X_{k}$ be the random variable indicating whether component $k$ is in a functioning $\left(X_{k}=1\right)$ or a failed $\left(X_{k}=0\right)$ state. Let $p_{k}=\operatorname{Pr}\left\{X_{k}=1\right\}$ be the probability that component $k$ functions, called the reliability of component $k$. Assume that $\mathbf{X}=\left(X_{1}, X_{2}, \ldots, X_{K}\right)$ are independent random variables, and let $\mathbf{p}=\left(p_{1}, p_{2}, \ldots, p_{K}\right)$ be the vector of components reliabilities. Under the assumption of independent components, the reliability function of a system with structure function $\phi$ is given by

$$
h_{\phi}(\mathbf{p})=E\{\phi(\mathbf{X})\}=\operatorname{Pr}\{\phi(\mathbf{X})=1\} .
$$


Thus, the reliability function of a series system is $h_{s e r}(\mathbf{p})=\prod_{j=1}^{K} p_{j}$, while that of a parallel system is $h_{\text {par }}(\mathbf{p})=1-\prod_{j=1}^{K}\left(1-p_{j}\right)$. On the other hand, the reliability function of the series-parallel system in Figure 1 is $h_{s p}\left(p_{1}, p_{2}, p_{3}\right)=p_{1}\left[1-\left(1-p_{2}\right)\left(1-p_{3}\right)\right]$. The reliability function for the more general $k$-out-of- $K$ system is

$$
h_{k: K}(\mathbf{p})=\sum_{\left\{\left(x_{1}, x_{2}, \ldots, x_{K}\right) \in\{0,1\}^{K} ; \sum_{j=1}^{K} x_{j} \geq k\right\}}\left[\prod_{j=1}^{K} p_{j}^{x_{j}}\left(1-p_{j}\right)^{1-x_{j}}\right] .
$$

These reliability functions represent the probabilities that the systems are functioning as a function of component reliabilities.

Let $\mathbf{T}=\left(T_{1}, T_{2}, \ldots, T_{K}\right)$ be the vector of component lifetimes and $S$ be the system lifetime. For a given time $t$, the state vector of components is denoted by $\mathbf{X}(t)=\left(I\left(T_{1}>t\right), I\left(T_{2}>\right.\right.$ $\left.t), \ldots, I\left(T_{K}>t\right)\right)$, hence the state of the system at time $t$ is given by $\phi(\mathbf{X}(t))$. As a result $\{S>$ $t\}=\{\phi(\mathbf{X}(t))=1\}$. Therefore, the system lifetime survivor function, also referred to as the system's reliability function, is given by

$$
\bar{F}_{\phi}(t)=\operatorname{Pr}\{S>t\}=\operatorname{Pr}\{\phi(\mathbf{X}(t))=1\}=E\{\phi(\mathbf{X}(t))\} .
$$

Denote the components lifetime survivor functions by

$$
\bar{F}_{j}(t)=E\left\{I\left\{T_{j}>t\right\}\right\}=\operatorname{Pr}\left\{T_{j}>t\right\}, j=1,2, \ldots, K .
$$

Under the assumption that the components lifetimes are independent, the system reliability function is given by

$$
\bar{F}_{\phi}(t)=h_{\phi}\left(\bar{F}_{1}(t), \bar{F}_{2}(t), \ldots, \bar{F}_{K}(t)\right) .
$$

Suppose we monitor $n$ identical systems with structure function $\phi$, with the $i$ th system observed over the time period $\left[0, \tau_{i}\right]$. The end-of-monitoring time $\tau_{i}$ could represent administrative time or some other termination time not related to the $i$ th system's components lifetimes. Let $S_{1}, S_{2}, \ldots, S_{n}$ denote the $n$ systems lifetimes and $\tau_{1}, \tau_{2}, \ldots, \tau_{n}$ denote the end of monitoring times. In observing these $n$ systems, not all the $S_{i}$ 's will be completely observed since some of them will be rightcensored by their $\tau_{i}$ 's. The random variables for the $n$ systems will therefore be

$$
(\mathbf{V}, \delta)=\left(\left(V_{1}, \delta_{1}\right),\left(V_{2}, \delta_{2}\right), \ldots,\left(V_{n}, \delta_{n}\right)\right),
$$

where $V_{i}=\min \left\{S_{i}, \tau_{i}\right\}$ and $\delta_{i}=I\left\{S_{i} \leq \tau_{i}\right\}$.

A nonparametric estimator of the system reliability function based on right-censored system lifetimes $(\mathbf{V}, \delta)$ is the Kaplan and Meier (1958) estimator, also known as the product-limit estimator (PLE), given by

$$
\widehat{R}_{P L E}(t)=\prod_{s \leq t}\left[1-\frac{\Delta N(s)}{Y(s)}\right]
$$

with the convention that $0 / 0=0, \prod$ means product-integral, and the processes $N=\{N(s): s \in$ $\Re\}$ and $Y=\{Y(s): s \in \Re\}$ are defined via

$$
N(s)=\sum_{i=1}^{n} I\left\{V_{i} \leq s ; \delta_{i}=1\right\} \quad \text { and } \quad Y(s)=\sum_{i=1}^{n} I\left\{V_{i} \geq s\right\}
$$


Doss et al. (1989) developed a PL-type estimator of the system reliability function when component failure times are available by exploiting the relationship given in 2.1) between system reliability and components reliabilities. The idea implemented in Doss et al. (1989) is to use the $j$ th component's right-censored data to estimate $\bar{F}_{j}, j=1,2, \ldots, K$, and then to plug-in these estimates in (2.1). In Doss et al. (1989)'s approach, each system is monitored until it failed so that they observed all the system failure times. However, as mentioned above, the $i$ th system lifetime could be right-censored by $\tau_{i}$, while its component lifetimes could be right-censored by $\tau_{i}$ or the system lifetime $S_{i}$. We assume that the end of monitoring times are either fixed or random.

Let $T_{i j}$ denote the lifetime of component $j$ for the $i$-th system. Define $Z_{i j}=\min \left\{T_{i j}, S_{i j}^{*}, \tau_{i}\right\}$ and $\delta_{i j}=I\left(T_{i j} \leq \min \left\{S_{i j}^{*}, \tau_{i}\right\}\right)$. The right-censoring variable for $T_{i j}$ involves $S_{i j}^{*}$, where $S_{i j}^{*}$ is the lifetime of the system obtained by $j$ th component deleted from the original system. Note that $S_{i j}^{*}$ is independent of $T_{i j}$. To give an example, consider the 3-component series-parallel system in Figure 1 The right-censoring variable for $T_{1}$ involves $S_{1}^{*}=\max \left\{T_{2}, T_{3}\right\}$, which is independent of $T_{1}$, while the right-censoring variables for $T_{2}$ and $T_{3}$ involve $S_{2}^{*}=\min \left\{T_{1}, T_{3}\right\}$ and $S_{3}^{*}=\min \left\{T_{1}, T_{2}\right\}$, respectively.

Let $F$ denote the system lifetime distribution. We assign a prior on $F$ which is a partition-based Dirichlet measure (PBDM) (Sethuraman and Hollander (2009)). This type of nonparametric prior measure will be reviewed in Section 3 . The posterior measure of $F$, given the right-censored system lifetimes, is also a PBDM. Under an integrated squared-error loss function, the Bayes estimator of $F$ is the mean of the posterior PBDM. In a similar fashion, by assigning independent PBDM prior on each of the components lifetime distribution functions, we obtain Bayes estimators of the reliability functions of each of the components, given the right-censored components data. A system reliability estimator, based on the components data, is then obtained by plugging-in these components reliability functions estimators in 2.1.

\section{Classes of Prior Probability Measures}

In the first part of this section we briefly review the general class of partition-based probability measures (PBPM) which was introduced in Sethuraman and Hollander (2009) in the context of making Bayesian statistical inference in repair models. We are using the class of PBDM prior because it is an elegant class of nonparametric conjugate prior for the incomplete observations such as lefttruncated, interval-censored, and right-censored data. In particular, these PBDM priors provides clear-cut (analytical) posterior calculation and we will provide a closed-form Bayes estimator as well as a procedure to sample from the posterior measure. These PBPMs will serve as prior probability measures on the relevant reliability functions of interest. The posterior probability measures, given the observed data, will also belong to this class of PBPMs. The PBPMs could be employed in a variety of settings with right-censored, interval-censored, and truncated data. For details regarding PBPMs we refer the reader to Sethuraman and Hollander (2009)'s paper. Let $(\mathscr{X}, \mathscr{A})$ be a measurable space and let $\mathscr{P}$ be the class of all probability measures (pms) on $(\mathscr{X}, \mathscr{A})$. Let $\mathscr{H}$ be the class of all pms on $(\mathscr{P}, \mathscr{S})$, where $\mathscr{S}=\sigma(\{P: P(A) \leq r, A \in \mathscr{A}, 0 \leq r \leq 1\})$. 
Definition 3.1. [Dirichlet Process (DP) (Ferguson (1973); Sethuraman (1994))]: Let $\alpha(\cdot)$ be a nonnull finite measure on $(\mathscr{X}, \mathscr{A})$. A random pm $P$ on $(\mathscr{P}, \mathscr{S})$ is said to be a Dirichlet process with parameter $\alpha(\cdot)$ if, for any measurable partition $\mathscr{B}=\left\{B_{1}, B_{2}, \ldots, B_{m}\right\}$ of $\mathscr{X}$, the distribution of $P(\mathscr{B}) \equiv\left(P\left(B_{1}\right), P\left(B_{2}\right), \ldots, P\left(B_{m}\right)\right)$ is the Dirichlet distribution $\mathscr{D}\left(\cdot ; \alpha\left(B_{1}\right), \alpha\left(B_{2}\right), \ldots, \alpha\left(B_{m}\right)\right)$. The pm in $\mathscr{H}$ governing such a $P$ will be denoted by $\mathscr{D}_{\alpha}$.

Definition 3.2. [Partition-Based Probability Measure (PBPM) (Sethuraman and Hollander (2009))]: Let $\mathscr{B}=\left(B_{1}, B_{2}, \ldots, B_{m}\right)$ be a measurable partition of $\mathscr{X} ; h:\left(\Re_{m}, \mathscr{B}_{m}\right) \rightarrow\left(\Re_{+}, \mathscr{B}_{+}\right)$taking the value zero outside of the $m$-dimensional simplex

$$
R^{m}=\left\{\mathbf{y}: y_{l} \geq 0, l=1, \ldots, m ; \sum_{l=1}^{m} y_{l}=1\right\}
$$

and with $\int_{R^{m}} h\left(y_{1}, \ldots, y_{m}\right) d y_{1} \cdots d y_{m}<\infty$. Let $G_{l}, l=1,2, \ldots, m$, be in $\mathscr{H}$ with $G_{l}$ having support that is a subset of $\left\{P \in \mathscr{P}: P\left(B_{l}\right)=1\right\}$, and let $\mathscr{G}$ be the product measure $G_{1} \times G_{2} \times$ $\cdots \times G_{m}$. A random pm $P \in \mathscr{P}$ is said to have a PBPM with parameters $(\mathscr{B}, h, \mathscr{G})$, denoted by $H \equiv H(\mathscr{B}, h, \mathscr{G}) \in \mathscr{H}$, if the following conditions are satisfied under $H$.

(a) $P(\mathscr{B}) \equiv\left(P\left(B_{1}\right), P\left(B_{2}\right), \ldots, P\left(B_{m}\right)\right)$ and the restricted random pms $P_{B_{l}}, l=1,2, \ldots, m$, are independent, where for any $P \in \mathscr{P}$, its restriction on a non-empty $B \in \mathscr{A}$ is given by

$$
P_{B}(A)=\frac{P(A \cap B)}{P(B)} I\{P(B)>0\}+I\{b \in A\} I\{P(B)=0\}
$$

with $b$ an arbitrary element of $B$;

(b) $P(\mathscr{B})=\left(P\left(B_{1}\right), P\left(B_{2}\right), \ldots, P\left(B_{m}\right)\right)$ has pdf $c h(\mathbf{y})$ where $c$ is a normalizing constant and $\mathbf{y} \in R^{m}$

(c) for each $l=1,2, \ldots, m, P_{B_{l}} \sim G_{l}$,

and, for each $A \in \mathscr{A}, P(A)=\sum_{l=1}^{m} P_{B_{l}}(A) P\left(B_{l}\right)$.

Definition 3.3. [Partition-Based Dirichlet Measure (PBDM)]: Let $\alpha(\cdot)$ be a finite non-null measure on $(\mathscr{X}, \mathscr{A})$. For any non-empty set $B \in \mathscr{A}$ with $\alpha(B)>0$, let $\alpha_{B}(A)=\alpha(A \cap B)$ for all $A \in \mathscr{A}$. Let $\mathscr{B}=\left(B_{1}, \ldots, B_{m}\right)$ be a measurable partition of $\mathscr{X}$ with $\alpha\left(B_{l}\right)>0, l=1, \ldots, m$. If $\mathscr{G}=\mathscr{D}_{\alpha_{B_{1}}} \times \ldots \times \mathscr{D}_{\alpha_{B_{m}}}$, then $H(\mathscr{B}, h, \mathscr{G}) \equiv \mathscr{D}(\mathscr{B}, h, \alpha)$ is called a Partition-Based Dirichlet Measure (PBDM).

An intermediate result in Sethuraman and Hollander (2009) also showed that the Dirichlet process $\mathscr{D}_{\alpha}$ coincides with $\mathscr{D}(\mathscr{B}, h, \alpha)$ for any partition $\mathscr{B}=\left(B_{1}, \ldots, B_{m}\right)$ where, with $\alpha_{l}=$ $\alpha\left(B_{l}\right), l=1,2, \ldots, m, h$ is of the form

$$
h(\mathbf{y})=\left[\frac{\Gamma\left(\sum_{l=1}^{m} \alpha_{l}\right)}{\prod_{l=1}^{m} \Gamma\left(\alpha_{l}\right)} \prod_{l=1}^{m} y_{l}^{\alpha_{l}-1}\right] I\left\{\mathbf{y} \in R^{m}\right\} .
$$


The PBPM will be used as the prior measure over the space of probability measures. Given the observed data, from the Bayesian inference perspective, it is of major importance to determine the posterior measure. Theorem 1 provides the initial result for this purpose. The proof of this result immediately follows from Theorem 3 in Sethuraman and Hollander (2009) hence will not be presented.

Theorem 1. Let $P \in \mathscr{P}$ have a PBPM prior probability measure $H(\mathscr{B}, h, \mathscr{G})$. Given $P$, let $T$ be an observation from $P$, so that $T \mid P \sim P$. Let $\mathscr{B}=\left(B_{1}, \ldots, B_{m}\right)$ be a measurable partition of $\mathscr{X}$ and $R \equiv R(T) \in\{1,2, \ldots, m\}$ be such that $T \in B_{R}$. Then, the posterior probability measure of $P$, given $T=t$ and with $R=r$, is the $P B P M H\left(\mathscr{B}, h^{*}, \mathscr{G}^{*}\right)$, where $h^{*}(\mathbf{y}) \propto h(\mathbf{y}) y_{r}$ and $\mathscr{G}^{*}=\times_{s \neq r} G_{s} \times G_{r}^{t}$, where $G_{s}^{t}$ is the posterior distribution of $P_{B_{s}}$, given $T_{s}=t$, where $T_{s} \mid P \sim P_{B_{s}}$ and $P_{B_{s}} \sim G_{s}$. In particular, if $H(\mathscr{B}, h, \mathscr{G})=\mathscr{D}(\mathscr{B}, h, \alpha)$, then the posterior is $\mathscr{D}\left(\mathscr{B}, h^{*}, \alpha^{*}\right)$ with $\alpha^{*}=\alpha+\delta_{t}$.

The preceding result pertains to the situation where a complete observation has been observed. The next result, Theorem 2, pertains to the posterior measure in light of a censored or incomplete observation. This follows from Theorem 1 in Grego et al. (2013) hence its proof will not also be presented.

Theorem 2. Let $P \in \mathscr{P}$ have a $P B P M$ prior probability measure $H(\mathscr{B}, h, \mathscr{G})$ and let $T \mid P \sim P$. Let $E \subset\{1,2, \ldots, m\}$ such that $T \in A=\cup_{j \in E} B_{j}$. Then, the posterior probability measure of $P$, given $T \in A$, is the PBPM $H\left(\mathscr{B}, h^{*}, \mathscr{G}\right)$, where $h^{*}(\mathbf{y}) \propto h(\mathbf{y}) y_{A}$ with $y_{A}=\sum_{j \in E} y_{j}$.

Theorem 2 is obtained when $A$ is a union of sets in the partition $\mathscr{B}$. When $A$ is not necessarily a union of sets in the partition $\mathscr{B}$, we can form a larger partition

$$
\mathscr{B}^{* *}=\left\{(\mathscr{B} \cap A) \cup\left(\mathscr{B} \cap A^{c}\right)\right\},
$$

which ensures that $A$ is a union of sets in the enlarged partition $\mathscr{B}^{* *}$. We could then start by having a PBPM $H\left(\mathscr{B}^{* *}, h^{* *}, \mathscr{G}^{* *}\right)$ that is appropriate for this enlarged partition.

Next, we consider the posterior distribution of $P$, whose prior distribution is a PBPM, when a right-censored data is available. Let $T_{1}, T_{2}, \ldots, T_{n}$ be a random sample from $P$ so that

$$
\left(T_{1}, T_{2}, \ldots, T_{n}\right) \mid P \stackrel{i i d}{\sim} P
$$

We are only able to observe the right-censored data $\left\{\left(V_{i}, \delta_{i}\right), i=1,2, \ldots, n\right\}$ where $\delta_{i}=1(0)$ means that $T_{i}=(>) V_{i}$. For such a right-censored data, define the subset of indices

$$
\mathscr{I}_{0}=\left\{i \in\{1,2, \ldots, n\}: \delta_{i}=0\right\} \quad \text { and } \quad \mathscr{I}_{1}=\left\{i \in\{1,2, \ldots, n\}: \delta_{i}=1\right\} .
$$

We shall consider a partition of $\mathscr{X}=\Re$ given by $\mathscr{B}=\left\{B_{s}: s=1,2, \ldots, m\right\}$ such that for each $i \in \mathscr{I}_{0}$ there exists an $E_{i} \subset\{1,2, \ldots, m\}$ satisfying

$$
\left(V_{i}, \infty\right)=\bigcup_{s \in E_{i}} B_{s}
$$

For each $i \in \mathscr{I}_{1}$, we let $r_{i} \in\{1,2, \ldots, m\}$ such that $V_{i} \in B_{r_{i}}$. With these notation, we are now in position to describe the posterior distribution of $P$, which is stated for general PBPMs. 
Theorem 3. Let $\left(T_{1}, T_{2}, \ldots, T_{n}\right) \mid P \stackrel{i \text { id }}{\sim} P$ and let $P$ have the PBPM prior distribution $H(\mathscr{B}, h, \mathscr{G})$ where $\mathscr{G}=\times{ }_{s=1}^{m} G_{s}$. Then, the posterior distribution of $P$ given the right-censored sample $\left\{\left(v_{i}, \delta_{i}\right)\right.$ : $i=1,2, \ldots, n\}$, and when the right-censoring mechanism is noninformative about $P$, is the PBPM $H\left(\mathscr{B}, h^{*}, \mathscr{G}^{*}\right)$, where

$$
\begin{aligned}
h^{*}(\mathbf{y}) & \propto h(\mathbf{y})\left[\prod_{i \in \mathscr{I}_{1}} y_{r_{i}}\right]\left[\prod_{i \in \mathscr{I}_{0}}\left(\sum_{j \in E_{i}} y_{j}\right)\right] \\
\mathscr{G}^{*} & =\times_{s=1}^{m} G_{s}^{\left(v_{i}: i \in \mathscr{I}_{1}, r_{i}=s\right)},
\end{aligned}
$$

with $G_{s}^{\left(t_{1}, \ldots, t_{k}\right)}$ the posterior distribution of $P_{B_{s}}$ when its prior is $G_{s}$ and given the observed data $T_{1}=t_{1}, \ldots, T_{k}=t_{k}$, where $\left(T_{1}, \ldots, T_{k}\right) \mid P \stackrel{\text { iid }}{\sim} P_{B_{s}}$, with the convention that $G_{s}^{\emptyset}=G_{s}$. In particular, if $H(\mathscr{B}, h, \mathscr{G})=\mathscr{D}(\mathscr{B}, h, \alpha)$, then the posterior distribution of $P$ given the right-censored data is $\mathscr{D}\left(\mathscr{B}, h^{*}, \alpha^{*}\right)$ with $\alpha^{*}=\alpha+\sum_{i \in \mathscr{I}_{1}} \delta_{v_{i}}$.

Proof. Repeated application of Theorem 1 for complete observations and repeated application of Theorem 2 for right-censored observations yield the stated result. However, a detail proof is given in the appendix when PBPM is partition-based Dirichlet measure (PBDM) $[H(\mathscr{B}, h, \mathscr{G})=$ $\mathscr{D}(\mathscr{B}, h, \alpha)]$.

\section{System Reliability Function Estimators}

\subsection{Based Only on System Data}

In this subsection we discuss inference concerning the system reliability function based only on the possibly right-censored system data. We assume that $n$ systems lifetimes, $S_{1}, S_{2}, \ldots, S_{n}$ are IID from an unknown distribution function $F$, or equivalently, an unknown probability measure $P$, so that $F(t)=P\{(-\infty, t]\}$ for each $t \in \Re$. Recall the random variables from $n$ systems are $\left(\left(V_{1}, \delta_{1}\right),\left(V_{2}, \delta_{2}\right), \ldots,\left(V_{n}, \delta_{n}\right)\right)$, where $V_{i}=\min \left(S_{i}, \tau_{i}\right)$ and $\delta_{i}=I\left(S_{i} \leq \tau_{i}\right)$ for $i=1,2, \ldots, n$. Furthermore, the noninformativeness of the right-censoring mechanism can be achieved by assuming that the distribution of the $\tau_{i}$ 's does not depend on $F$ or $P$.

The main goal is to develop a nonparametric Bayes estimator of $F$ or $P$ based on the rightcensored system data when a PBDM prior is assigned on $P$. The prior measure on $P$ is the PBDM $\mathscr{D}(\mathscr{B}, h, \alpha)$. Theorem 3 provides the posterior distribution of $P$ given the right-censored systems data. For an estimate $\hat{F}$ of $F$, its quality will be measured via the integrated squared-error loss function

$$
L(F, \hat{F})=\int_{0}^{\infty}[\hat{F}(t)-F(t)]^{2} d t .
$$

With respect to this loss function, the Bayes estimator of $F$, which minimizes the Bayes risk, is given by the posterior mean of $F$ given the right-censored data., that is,

$$
\hat{F}_{B}(t)=E\left\{F(t) \mid\left\{\left(V_{i}, \delta_{i}\right), i=1,2, \ldots, n\right\}\right\} .
$$


We shall denote by $\hat{P}_{B}(\cdot)$ the probability measure induced by $\hat{F}_{B}(\cdot)$, which will be the Bayes estimator of the probability measure $P(\cdot)$. In order to obtain closed-form expressions and to relate to the product-limit estimator, we shall obtain this Bayes estimator under the PBPM prior $\mathscr{D}(\mathscr{B}, h, \alpha)$ with $h$ being of specific form

$$
h(\mathbf{y}) \propto\left[\prod_{l=1}^{m} y^{\alpha_{l}-1}\right] I\left\{\mathbf{y} \in R^{m}\right\},
$$

with $\alpha_{l}=\alpha\left(B_{l}\right), l=1,2, \ldots, m$. In addition, the partition $\mathscr{B}=\left(B_{1}, B_{2}, \ldots, B_{m}\right)$ of $\Re$ will be such that each $B_{l}$ is an interval and such that $B_{l}<B_{l+1}$ for each $l=1,2, \ldots, m-1$. We also introduce the following notations. For $l=1,2, \ldots, m$ and $A \subset \Re$,

$$
\begin{gathered}
N_{l}(A)=\sum_{i \in \mathscr{I}_{1}} I\left\{V_{i} \in B_{l} \cap A\right\} \quad \text { and } \quad N_{l}=N_{l}\left(B_{l}\right) ; \\
J_{l}=\sum_{i \in \mathscr{I}_{0}} I\left\{V_{i} \in B_{l}\right\} ; \\
N_{\geq l}(A)=\sum_{j=l}^{m} N_{j}(A) ; \quad N_{\geq l}=N_{\geq l}\left(B_{l}\right) ; \quad \text { and } \quad J_{\geq l}=\sum_{j=l}^{m} J_{j} .
\end{gathered}
$$

Theorem 4. The Bayes estimator of $P$ under integrated squared-error loss function when the prior of $P$ is a $\mathscr{D}(\mathscr{B}, h, \alpha)$ with $h$ of form in (4.1) and when given the right-censored systems data is

$$
\hat{P}(A)=\sum_{k=1}^{m} W_{k} \hat{P}_{k}(A)
$$

where, for $k=1,2, \ldots, m$,

$$
W_{k}=\left\{\left[\frac{\alpha_{k}+N_{k}}{\alpha_{\geq k}+N_{\geq k}+J_{\geq k+1}}\right]^{I\{k \leq m-1\}}\right\}\left\{\prod_{l=1}^{k-1}\left[\frac{\alpha_{\geq l+1}+N_{\geq l+1}+J_{\geq l+1}}{\alpha_{\geq l}+N_{\geq l}+J_{\geq l+1}}\right]\right\} ;
$$

and

$$
\hat{P}_{k}(A)=\frac{\alpha\left(B_{k} \cap A\right)+N_{k}(A)}{\alpha\left(B_{k}\right)+N_{k}} .
$$

For clarity, we point out that the first term in braces of the expression for $W_{m}$ is equal to 1 . Thus, observe that the Bayes estimator of $P$ is a mixture of the Bayes estimators, under Dirichlet priors, of the restricted probability measures at each of the members of the partition. The mixing weights are dependent on the prior $\alpha$ and the information from the right-censored systems data on each of the partition members.

Prior to proving this result, we point out two special cases of this estimator. The first case is when there are no right-censored observations, then we recover the usual Bayes estimator; while the second case is when $\alpha(\Re) \rightarrow 0$ and there are right-censored values. In this latter case we do not immediately obtain the product-limit estimator, but if we further refine the partition to include the singleton sets determined by the observed failure times, then we do recover the PLE as a special case. 
Corollary 1. If there are no right-censored observations, then

$$
\hat{P}(A)=\frac{\sum_{k=1}^{m}\left[\alpha_{k}(A)+N_{k}(A)\right.}{\alpha(\Re)+n}=\frac{\alpha(A)+N(A)}{\alpha(\Re)+n},
$$

where $N(A)=\sum_{i=1}^{n} I\left\{V_{i} \in A\right\}=\sum_{i=1}^{n} I\left\{T_{i} \in A\right\}$. As such, when $\alpha(\Re) \rightarrow 0, \hat{P}(A)$ converges to the empirical measure of $A: N(A) / n$.

Corollary 2. Let $V_{(1)}<V_{(2)}<\ldots<V_{(K)}$ denote the distinct observed failure times, and let $R_{(k)}$ be such that $V_{(k)} \in B_{R_{(k)}}$. Then, when $\alpha(\Re) \rightarrow 0$, we obtain

$$
\hat{P}\left(\left\{V_{(k)}\right\}\right) \rightarrow\left\{\begin{array}{cl}
{\left[\frac{N_{R_{(k)}}\left(\left\{V_{(k)}\right\}\right)}{N_{\geq R_{(k)}}+J_{\geq R_{(k)}}+1}\right]\left[\prod_{l=1}^{R_{(k)}-1}\left(1-\frac{N_{l}}{N_{\geq l}+J_{\geq l+1}}\right)\right]} & \text { if } \quad R_{(k)}<m \\
{\left[\frac{N_{m}\left(\left\{V_{(k)}\right\}\right)}{N_{m}}\right]\left[\prod_{l=1}^{m-1}\left(1-\frac{N_{l}}{N_{\geq l}+J_{\geq l+1}}\right)\right]} & \text { if } \quad R_{(k)}=m
\end{array} .\right.
$$

This limit estimator is not yet the PLE. However, if we include in the partition the singleton sets $\left\{V_{(k)}\right\}, k=1,2, \ldots, K$, and we let $D_{(k)}$ be the number of failures at $V_{(k)}$ and $Y_{(k)}$ be the number-at-risk at $V_{(k)}$, then as $\alpha(\Re) \rightarrow 0$, we obtain

$$
\hat{P}\left(\left\{V_{(k)}\right\}\right) \rightarrow\left\{\begin{array}{ccc}
{\left[\frac{D_{(k)}}{Y_{(k)}}\right]\left[\prod_{l=1}^{k-1}\left(1-\frac{D_{(l)}}{Y_{(l)}}\right)\right]} & \text { if } & k<K \\
{\left[\prod_{l=1}^{K-1}\left(1-\frac{D_{(l)}}{Y_{(l)}}\right)\right]} & \text { if } & k=K
\end{array},\right.
$$

which coincides with a version of the PLE. Observe that the number of failures and the number-atrisk at $V_{(k)}$ are, for $k=1,2, \ldots, K$,

$$
\begin{aligned}
& D_{(k)}=\sum_{i=1}^{n} I\left\{V_{i}=V_{(k)}, \delta_{i}=1\right\} \\
& Y_{(k)}=N_{\geq R_{(k)}}+Y_{\geq R_{(k)}+1}=N_{R_{(k)}}+\sum_{l=R_{(k)}+1}^{m}\left(N_{l}+J_{l}\right),
\end{aligned}
$$

and the estimator $\hat{P}$ has atoms only at the $V_{(k)}$ 's.

We now establish Theorem 4 We shall do this in steps.

Lemma 4.1. For every $m=2,3, \ldots$, and $\alpha=\left(\alpha_{1}, \ldots, \alpha_{m}\right) \in \Re_{+}^{m}$ and $\beta=\left(\beta_{1}, \ldots, \beta_{m}\right) \in \Re_{+, 0}^{m}$, with $R^{m}=\left\{\mathbf{y}=\left(y_{1}, \ldots, y_{m}\right) \in(0,1)^{m}: y_{m}=1-\sum_{i=1}^{m-1} y_{i}\right\}$,

$$
C_{m}(\alpha, \beta) \equiv \int_{R^{m}} \prod_{l=1}^{m}\left[y_{l}^{\alpha_{l}-1}\left(\sum_{j=l}^{m-1} y_{j+1}\right)^{\beta_{l}}\right] d \mathbf{y}=\prod_{l=1}^{m-1} B\left(\alpha_{l}, \alpha_{\geq l+1}+\beta_{\geq l+1}\right),
$$

where $B(\alpha, \beta)=\Gamma(\alpha) \Gamma(\beta) / \Gamma(\alpha+\beta)$ is the beta function.

Proof. This result can be straightforwardly established using mathematical induction over $m$ starting with $m=2$, hence we leave the details of the proof to the reader. 
Lemma 4.2. Let $\mathbf{Y}=\left(Y_{1}, Y_{2}, \ldots, Y_{m}\right)$ have probability density function

$$
h(\mathbf{y}) \propto \prod_{l=1}^{m}\left[y_{l}^{\alpha_{l}-1}\left(\sum_{j=l}^{m-1} y_{j+1}\right)^{\beta_{l}}\right] I\left\{\mathbf{y} \in R^{m}\right\} .
$$

Then, with the notation $\left(\alpha, a_{k}\right)=\left(\alpha_{1}, \ldots, \alpha_{k-1}, \alpha_{k}+a_{k}, \alpha_{k+1}, \ldots, \alpha_{m}\right)$, for $k=1,2, \ldots, m$,

$$
E\left[Y_{k}\right]=\frac{C\left(\left(\alpha, 1_{k}\right), \beta\right)}{C(\alpha, \beta)}=\left[\frac{\alpha_{k}}{\alpha_{\geq k}+\beta_{\geq k+1}}\right]^{I\{k<m\}} \prod_{l=1}^{k-1}\left[\frac{\alpha_{\geq l+1}+\beta_{\geq l+1}}{\alpha_{\geq l}+\beta_{\geq l+1}}\right] .
$$

Proof . Straight-forward simplifications using properties of the beta and gamma functions, hence we do not present details of the proof.

Proof of Theorem 4; Given the DATA $=\left\{\left(V_{i}, \delta_{i}\right), i=1,2, \ldots, n\right\}$, the right-censored systems data, the posterior distribution of $P$ is $\mathscr{D}\left(\mathscr{B}, h^{*}, \alpha^{*}\right)$ where $\alpha^{*}(B)=\alpha(B)+\sum_{i \in \mathscr{I}_{1}} I\left\{v_{i} \in B\right\}$ and

$$
h^{*}(\mathbf{y}) \propto h(\mathbf{y})\left[\prod_{i \in \mathscr{I}_{1}} y_{r_{i}}\right]\left[\prod_{i \in \mathscr{I}_{0}}\left(\sum_{j \in E_{i}} y_{j}\right)\right]
$$

where, for $i \in \mathscr{I}_{0},\left(V_{i}, \infty\right)=\cup_{j \in E_{i}} B_{j}$. Let $J_{l}, l=1,2, \ldots, m$ be such that

$$
J_{l}=\sum_{i \in \mathscr{I}_{0}} I\left\{\left(v_{i}, \infty\right)=\cup_{j=l}^{m} B_{j}\right\} .
$$

Then, using the form of $h$ given in 4.1, we have that

$$
h^{*}(\mathbf{y}) \propto \prod_{l=1}^{m}\left\{y_{l}^{\alpha_{l}+N_{l}-1}\left(\sum_{j=l}^{m} y_{j}\right)^{J_{l}}\right\} I\left\{\mathbf{y} \in R^{m}\right\} .
$$

Consequently, by properties of PBPM, the Bayes estimator of $P(A)$ is given by

$$
\hat{P}(A)=E\{P(A) \mid \mathrm{DATA}\}=\sum_{l=1}^{m} E\left\{P\left(B_{l}\right) \mid \mathrm{DATA}\right\} E\left\{P_{B_{l}}(A) \mid \mathrm{DATA}\right\} .
$$

Since $P_{B_{l}} \mid$ DATA $\sim \mathscr{D}\left(\alpha^{*}\left(B_{l} \cap \cdot\right)\right)$, it follows from properties of the Dirichlet process that

$$
\begin{aligned}
& E\left\{P_{B_{l}}(A) \mid \text { DATA }\right\}=\frac{\alpha^{*}\left(B_{l} \cap A\right)}{\alpha^{*}\left(B_{l}\right)} \\
& =\frac{\alpha\left(B_{l} \cap A\right)+\sum_{i \in \mathscr{I}_{1}} I\left\{V_{i} \in B_{l} \cap A\right\}}{\alpha\left(B_{l}\right)+\sum_{i \in \mathscr{I}_{1}} I\left\{V_{i} \in B_{l}\right\}}=\frac{\alpha_{l}(A)+N_{l}(A)}{\alpha_{l}+N_{l}} .
\end{aligned}
$$


On the other hand, the density function of $\left(P\left(B_{1}\right), P\left(B_{2}\right), \ldots, P\left(B_{m}\right)\right)$, given DATA, is $h^{*}(\mathbf{y})$. Consequently,

$$
\begin{aligned}
E\left\{P\left(B_{k}\right) \mid \mathrm{DATA}\right\} & =E^{h^{*}}\left[Y_{k}\right] \\
& =\frac{\int_{R^{m}} y_{k} h^{*}(\mathbf{y}) d \mathbf{y}}{\int_{R^{m}} h^{*}(\mathbf{y}) d \mathbf{y}} \\
& =\frac{C\left(\left(\alpha+\mathbf{N}, 1_{k}\right), \mathbf{J}\right)}{C(\alpha+\mathbf{N}, \mathbf{J})} \\
& =\left[\frac{\alpha_{k}+N_{k}}{\alpha_{\geq k}+N_{\geq k}+J_{\geq k+1}}\right]^{I\{k<m\}} \prod_{l=1}^{k-1}\left[\frac{\alpha_{\geq l+1}+N_{\geq l+1}+J_{\geq l+1}}{\alpha_{\geq l}+N_{\geq l}+J_{\geq l+1}}\right],
\end{aligned}
$$

with the last equality obtained by using Lemma 4.2 . Combining these results lead to the expression for $\hat{P}(A)$, completes the proof of Theorem 4

\subsection{Pointwise Credible Intervals}

To construct pointwise credible intervals for $\bar{F}(t)=1-F(t)=P((t, \infty))$, we take samples from the posterior measure given in 4.3 . That is, the density function associated with the posterior measure is given by

$$
h^{*}(\mathbf{y}) \propto \prod_{l=1}^{m}\left\{y_{l}^{\alpha_{l}+N_{l}-1}\left(\sum_{j=l}^{m} y_{j}\right)^{J_{l}}\right\} I\left\{\mathbf{y} \in R^{m}\right\}
$$

which is also proportional to the so-called generalized Dirichlet distribution (see Connor and Mosimann (1969)). To sample from the posterior measure, we consider a well-known transformations

$$
X_{l}=Y_{l}+Y_{l+1}+\ldots+Y_{m}, l=1,2, \ldots, m \text {. }
$$

Define,

$$
W_{l}=\frac{X_{l+1}}{X_{l}}, l=1,2, \ldots, m-1
$$

Simplification yields that

$$
Y_{1}=1-W_{1}, Y_{2}=W_{1}\left(1-W_{2}\right), \ldots, Y_{m-1}=\left(1-W_{m-1}\right) \prod_{j=1}^{m-2} W_{j}, Y_{m}=\prod_{j=1}^{m-1} W_{j} .
$$

Straight-forward derivations show that $W_{1}, W_{2}, \ldots, W_{m-1}$ have independent beta distributions with

$$
W_{1} \sim \operatorname{Beta}\left(A_{1}, \alpha_{1}^{*}\right), W_{2} \sim \operatorname{Beta}\left(A_{2}, \alpha_{2}^{*}\right), \ldots, W_{m-1} \sim \operatorname{Beta}\left(A_{m-1}, \alpha_{m-1}^{*}\right),
$$

where $A_{r}=\sum_{l=r}^{m}\left[\alpha_{l}+N_{l}+J_{l}\right]$ and $\alpha_{r}^{*}=\alpha_{r}+N_{r}$, for $r=1,2, \ldots, m$. One may now take samples of $W_{1}, W_{2}, \ldots, W_{m}$, and then obtain $Y_{1}, Y_{2}, \ldots, Y_{m}$ using (4.4). Then, for any $t \in B_{l} \subseteq \mathscr{B}$, an 
approximate posterior mean, hence an approximate nonparametric Bayes estimator, is given by

$$
\hat{F}_{N P B}(t)=\hat{P}((0, t])=\sum_{j=1}^{l-1} E\left(Y_{j}\right)+E\left(Y_{l}\right)\left[\frac{\alpha\left(B_{l} \cap(0, t]\right)+N_{l}\left(B_{l} \cap(0, t)\right)}{\alpha\left(B_{l}\right)+N_{l}}\right]
$$

could be obtained and point-wise credible intervals of $F_{N P B}(t)\left(\right.$ or $\left.\bar{F}_{N P B}(t)=1-F_{N P B}(t)\right)$ could be constructed from the posterior samples.

\subsection{Joint Estimation of System and Components Reliabilities}

We now consider the joint estimation of component and system reliabilities when $n$ identical systems each with $K$ components are in the study. Denote the lifetime of component $j$ in the $i$-th system by $\left\{T_{i j}\right\}$ and let $\left(0, \tau_{i}\right]$ be the monitoring period for the $i$-th system. Assume that, for each $j=$ $1, \ldots, K,\left\{T_{i j}, i=1,2 \ldots, n\right\}$ are IID with distribution $F_{j}$, and $\left\{T_{i j}\right\} \mathrm{s}$ and $\left\{\tau_{i}\right\} \mathrm{s}$ are independent, $T_{i j}$ and $T_{i l}$ are also independent for $j \neq l$. Recall that the random observables for the $j$-th component are

$$
\left\{\left(Z_{i j}, \delta_{i j}\right), i=1,2, \ldots, n\right\}, j=1,2, \ldots, K .
$$

We assign independent PBDM prior on $P_{j}, j=1,2, \ldots, K$, and obtain nonparametric Bayes estimators of $P_{j}, j=1,2, \ldots, K$, given the random observable $\left\{\left(Z_{i j}, \delta_{i j}\right), i=1,2, \ldots, n\right\}$ for the $j$-th component. For such right-censored components data we define the following subset of indices

$$
\mathscr{I}_{0 j}=\left\{i \in\{1,2, \ldots, n\}: \delta_{i j}=0\right\} \quad \text { and } \quad \mathscr{I}_{1 j}=\left\{i \in\{1,2, \ldots, n\}: \delta_{i j}=1\right\} .
$$

Without loss of generality we consider a partition of $\mathscr{X}=\Re$ given by $\mathscr{B}=\left\{B_{s}: s=1,2, \ldots, m\right\}$ such that for each component $j$ and for each $i \in \mathscr{I}_{0 j}$ there exists an $E_{i j} \subset\{1,2, \ldots, m\}$ satisfying

$$
\left(Z_{i j}, \infty\right)=\bigcup_{s \in E_{i j}} B_{s}
$$

Define, $P_{j}\left(B_{l}\right)=Y_{l j}$. Assume that the random probability measure $P_{j}$ has PBDM prior measure, $\mathscr{D}\left(\mathscr{B}, h_{j}, \alpha_{j}\right)$, where $\alpha_{j}=\left(\alpha_{1 j}, \alpha_{2 j}, \ldots, \alpha_{m j}\right)$ with $\alpha_{l j}=\alpha_{j}\left(B_{l}\right), l=1,2, \ldots, m$, and $h_{j} \equiv$ $h\left(\mathbf{y}_{j}\right)$ is given by

$$
h\left(\mathbf{y}_{j}\right) \propto\left[\prod_{l=1}^{m} y_{l j}^{\alpha_{l j}-1}\right] I\left\{\mathbf{y}_{j} \in R^{m}\right\},
$$

We also introduce the following notations. For $l=1,2, \ldots, m$ and $A \subset \Re$,

$$
\begin{gathered}
N_{l j}(A)=\sum_{i \in \mathscr{I}_{1 j}} I\left\{Z_{i j} \in B_{l} \cap A\right\} \quad \text { and } \quad N_{l j}=N_{l j}\left(B_{l}\right) ; \\
J_{l j}=\sum_{i \in \mathscr{I}_{0 j}} I\left\{Z_{i j} \in B_{l}\right\} ; \\
N_{\geq l}^{(j)}(A)=\sum_{r=l}^{m} N_{r j}(A) ; \quad N_{\geq l}^{(j)}=N_{\geq l}^{(j)}\left(B_{l}\right) ; \quad \text { and } \quad J_{\geq l}^{(j)}=\sum_{r=l}^{m} J_{r j} .
\end{gathered}
$$

Applying Theorem 4, we can then obtain a closed form estimator of $P_{j}, j=1,2, \ldots, K$, which is stated as Corollary 3. 
Corollary 3. The Bayes estimator of $P_{j}$ under integrated squared-error loss function when the prior of $P_{j}$ is a $\mathscr{D}\left(\mathscr{B}, h_{j}, \alpha_{j}\right)$ with $h_{j}$ of form in 4.5 and when given the right-censored components data is

$$
\hat{P}_{j}(A)=\sum_{r=1}^{m} W_{r j} \hat{P}_{r j}(A), \quad j=1,2, \ldots, K,
$$

where, for $r=1,2, \ldots, m$,

$$
W_{r j}=\left\{\left[\frac{\alpha_{r j}+N_{r j}}{\alpha_{\geq r}^{(j)}+N_{\geq r}^{(j)}+J_{\geq r+1}^{(j)}}\right]^{I\{r \leq m-1\}}\right\}\left\{\prod_{l=1}^{r-1}\left[\frac{\alpha_{\geq l+1}^{(j)}+N_{\geq l+1}^{(j)}+J_{\geq l+1}^{(j)}}{\alpha_{\geq l}^{(j)}+N_{\geq l}^{(j)}+J_{\geq l+1}^{(j)}}\right]\right\} ;
$$

and

$$
\hat{P}_{r j}(A)=\frac{\alpha_{j}\left(B_{r} \cap A\right)+N_{r}^{(j)}(A)}{\alpha_{j}\left(B_{r}\right)+N_{r}^{(j)}} .
$$

As pointed out earlier the first term in braces of the expression for $W_{m j}, j=1,2, \ldots, K$, is equal to 1 . Therefore a closed form estimate of the $j$-th components reliability function is given by $\widehat{\bar{F}}_{j}(t)=1-\widehat{F}_{j}(t)=\widehat{P}_{j}((t, \infty)), j=1,2, \ldots, K$. As a consequence an estimate of the system reliability function can be expressed in terms of the components reliability function using 2.1) and is given by

$$
\widehat{\bar{F}}_{\phi}(t)=h_{\phi}\left(\hat{\bar{F}}_{1}(t), \hat{\bar{F}}_{2}(t), \ldots, \hat{\overline{F_{K}}}(t)\right) .
$$

Denote the corresponding Doss et al. (1989) PL-type estimator of system reliability function by

$$
\widehat{R}_{\phi}(t)=h_{\phi}\left(\widehat{R}_{1, P L E}(t), \widehat{R}_{2, P L E}(t), \ldots, \widehat{R}_{K, P L E}(t)\right),
$$

where $\widehat{R}_{j, P L E}(t), j=1,2, \ldots, K$, are the PL-type estimators of the component reliability functions as defined in (2.3). That Doss et al. (1989) estimator (4.7) is a limiting case of our estimator (4.6) when the prior measures satisfy $\alpha_{j}\left(\Re^{+}\right) \rightarrow 0$ for $j=1,2, \ldots, K$, is formally stated in Corollary 4 which follows from Corollary 2

Corollary 4. Under the conditions of Corollary 3 when, for each $j=1, \ldots, K$, we have $\alpha_{j}\left(R^{+}\right) \rightarrow$ 0 , then $\widehat{\bar{F}}_{\phi}(t) \rightarrow \widehat{R}_{\phi}(t)$.

\section{Simulation Studies}

Simulation studies are carried out to examine the biases and root-mean-squared errors (RMSEs) of the nonparametric Bayes estimator of system reliability function based on system lifetime data, denoted by $\widehat{\bar{F}}_{N P B}(t)$ (labeled BayesSys), and components lifetimes data, denoted by $\widehat{\bar{F}}_{\phi}(t)$ (labeled BayesPhi), as well as corresponding nonparametric (PL-type) estimators denoted by $\widehat{R}_{P L E}(t)$ (labeled PLESys) and $\widehat{R}_{\phi}(t)$ (labeled PLEPhi). We consider the three component series-parallel system (Figure 11 with component lifetimes, $T_{i j} \sim \operatorname{Exp}\left(\theta_{j}\right), j=1,2,3 ; \theta=(1,2,1.5)$, and monitoring time $\tau_{i} \sim \operatorname{Exp}(1)$. Simulated biases and RMSEs are obtained at the 5th, 10th , ., 95th percentile of the true data generating distribution based on 1000 replications for $n=30$, and compare 

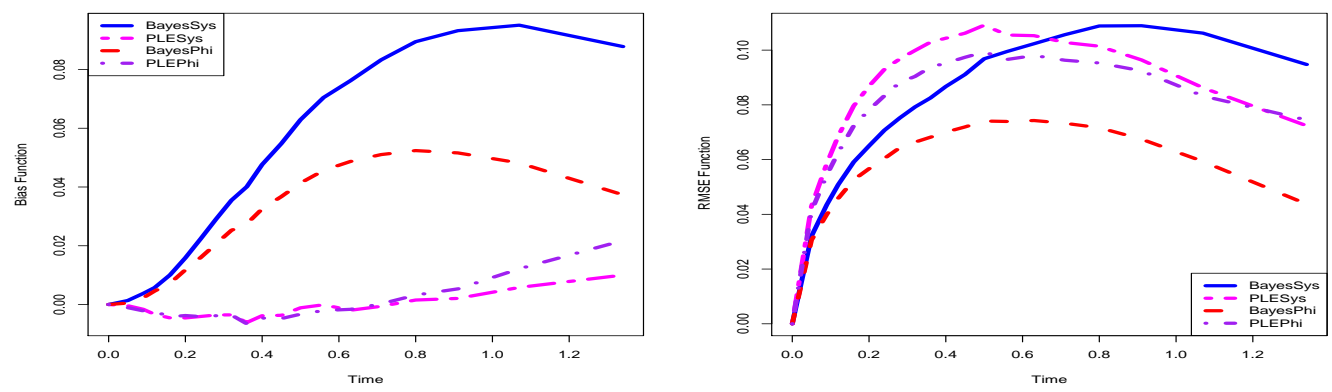

Figure 2: Simulated biases and RMSEs of the estimators $\widehat{\bar{F}}_{N P B}(t)$ (labeled BayesSys), $\widehat{\bar{F}}_{\phi}(t)$ (labeled BayesPhi), $\widehat{R}_{P L E}(t)$ (labeled PLESys), and $\widehat{R}_{\phi}(t)$ (labeled PLEPhi). Simulation parameters are $n=30$, lifetimes (3-component series-parallel system) from Exponential distribution with rate $\theta=(1,2,1.5)$, and with 1,000 replications.

those with the corresponding nonparametric estimators. To compute $\widehat{\bar{F}}_{\phi}(t)$ we assign independent PBDM prior measures with parameters $\alpha_{j}(t, \infty)=\beta_{j} \exp \left(-\theta_{j} t\right)$, where $\theta_{1}=1, \theta_{2}=2, \theta_{3}=1.5$ and $\beta_{j}=10, j=1,2,3$, on the components distribution functions $F_{j}, j=1,2,3$, respectively. That is, each PBDM prior measure has parameter that is $\beta_{j}$ multiplied by an exponential survivor function with parameter $\theta_{j}$. The $\beta_{j}$ s may be viewed as the degrees of precision of the PBDM prior measures. Note that in this case the prior mean functions, $\bar{\alpha}_{j}$ coincide with the components true reliability functions $\bar{F}_{j}, j=1,2,3$. To compute $\widehat{\bar{F}}_{N P B}(t)$ we also assign similar prior measure with $\theta=1$ and $\beta=1$. In this case the prior mean function does not coincide with the true distribution of system reliability. Even for the simple case, when components distributions are all exponentials, the system lifetime distribution is not an exponential distribution in this series-parallel system.

Figure 2 demonstrates that both $\widehat{\bar{F}}_{N P B}(t)$ and $\widehat{\bar{F}}_{\phi}(t)$ possess larger biases but smaller RMSEs than the nonparametric estimators $\widehat{R}_{P L E}$ and $\widehat{R}_{\phi}$, respectively. By examining Figure 2 it is evident that $\widehat{\bar{F}}_{\phi}$ has smaller biases and RMSEs than $\widehat{\bar{F}}_{N P B}(t)$, while $\widehat{R}_{P L E}$ exhibits slightly larger biases 


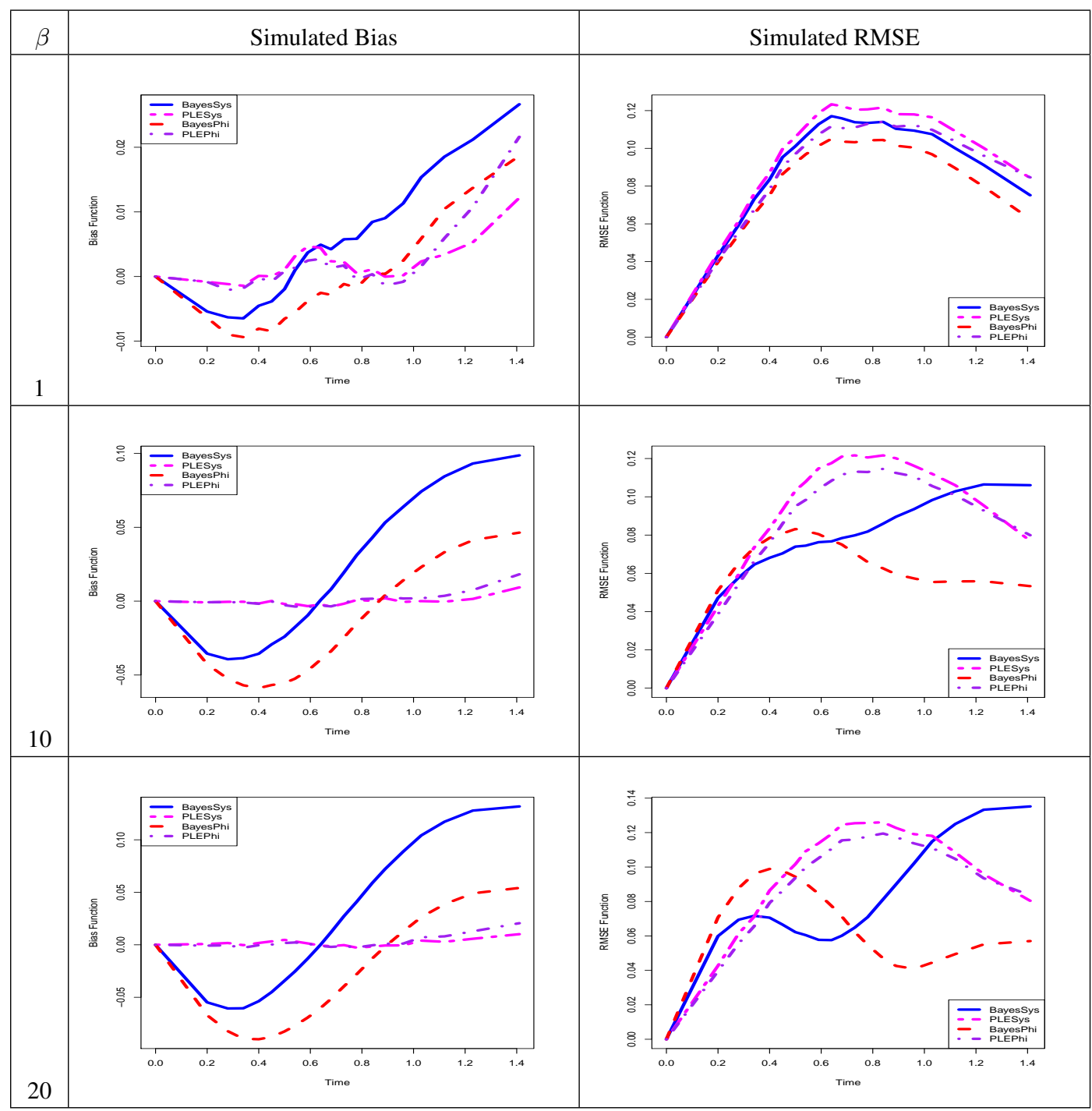

Figure 3: Simulated biases and RMSEs of the estimators $\widehat{\bar{F}}_{N P B}(t)$ (labeled BayesSys), $\widehat{\bar{F}}_{\phi}(t)$ (labeled BayesPhi), $\widehat{R}_{P L E}(t)$ (labeled PLESys), and $\widehat{R}_{\phi}(t)$ (labeled PLEPhi). Simulation parameters are $n=30$, $\theta=(1,1,1)$ and $\gamma=(2,1.5,1.2)$ (Weibull $(\theta, \gamma))$ with 1000 replications. Mis-specified PBDM prior measures with $\alpha(u, \infty)=\beta \exp (-\theta u)$, where $\theta=(1,1,1)$ and $\beta=(1,1,1),(10,10,10),(20,20,20)$.

and RMSEs than $\widehat{R}_{\phi}$. Among all four estimators, $\widehat{\bar{F}}_{\phi}(t)$ possesses the smallest RMSEs. Therefore the proposed estimator of the system reliability function, $\widehat{\bar{F}}_{\phi}$, based on the components lifetimes data, has the best performance. However, in practice it is unlikely that the mean of the PBDM prior measure will coincide with the true distribution function. So we carried out another simulation 
studies where the mean of the PBDM prior measures are misspecified. We also investigated the

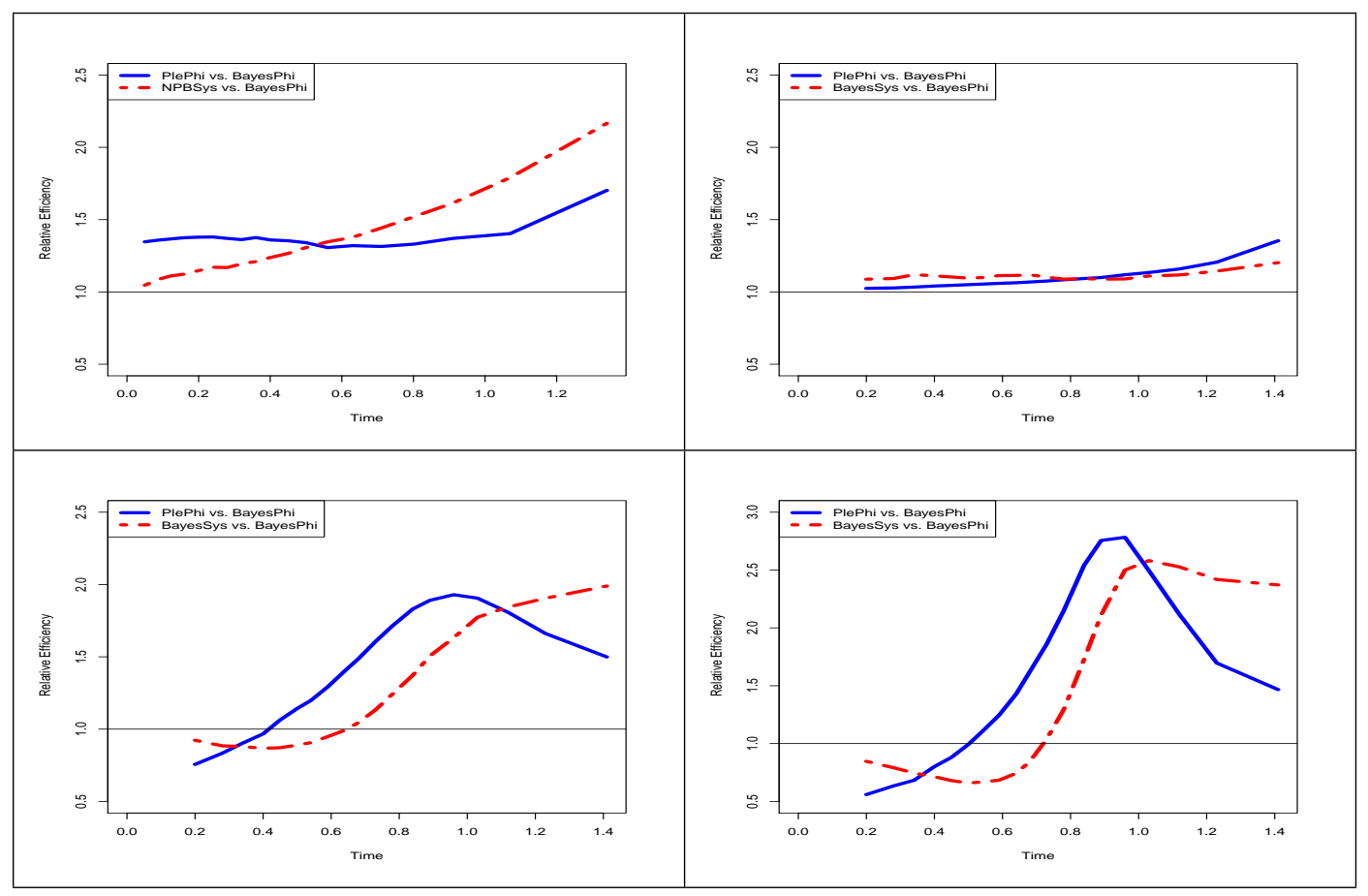

Figure 4: Asymptotic relative efficiency: true prior mean (top left), misspecified prior mean with $\beta=1$ (top right), $\beta=10$ (bottom left), and $\beta=20$ (bottom right).

biases and RMSEs in the case of misspecified prior measures, that is, when $\bar{\alpha}_{j}$ differ from the components true lifetimes generating distributions $\bar{F}_{j}, j=1,2,3$. In particular, for each component, IID lifetimes are generated from the Weibull distribution with a scale parameter $\theta_{j}$ and a shape parameter $\gamma_{j}$ with $\theta=(1,1,1), \gamma=(2,1.5,1.2)$ and random monitoring $\tau_{i} \sim \operatorname{Exp}(1)$. However, we assign the parameter of the PBDM prior measure to be $\alpha_{j}(t, \infty)=\beta_{j} \exp \left[-\theta_{j} t\right]$ instead of $\alpha_{j}(t, \infty)=\beta_{j} \exp \left[-\left(\theta_{j} t\right)^{\gamma_{j}}\right]$ with $\theta=(1,1,1)$, and $\beta=(1,1,1)$, so that $\bar{\alpha}_{j}$ is proportional to an exponential survivor function and thus the prior mean function differs from the true data generating distribution for each of the components. Aside from the choice of $\beta=1$, we also run the simulations for the cases $\beta \in\{(10,10,10),(20,20,20)\}$ to examine the effects of precision parameter $\beta$ when the PBDM priors are misspecified.

From Figure 3 , it is evident that for a smaller value of precision parameter, namely $\beta=(1,1,1)$, $\widehat{\bar{F}}_{\phi}(t)$ has smaller RMSE than $\widehat{R}_{\phi}(t)$ even in the case of misspecified prior measures. As the precision of the prior measure $\beta$ increases, $\widehat{\bar{F}}_{\phi}(t)$ possesses higher biases but smaller RMSE's than $\widehat{R}_{\phi}(t)$ except for smaller values of $t$. Figure 3 therefore indicates that the nonparametric Bayes estimator $\widehat{\bar{F}}_{\phi}(t)$ is robust in the sense that they do not suffer significantly due to a misspecification of the 
prior measures. When prior measures are misspecified, a larger magnitude of the precision parameter $\beta$ produces larger biases and RMSE's for the Bayes estimators $\widehat{\bar{F}}_{\phi}$ and $\widehat{\bar{F}}_{N P B}$. The negative impact of prior misspecification can be partly restrained by choosing smaller values of the precision parameters.

Relative efficiencies of different type of estimators of system reliability function are obtained. Relative efficiency of $\bar{F}_{N P B}(t)$ and $\bar{F}_{\phi}(t)$ is defined by $\frac{\operatorname{Var}\left(\bar{F}_{N P B}(t)\right)}{\operatorname{Var}\left(\bar{F}_{\phi}(t)\right)}$. Similarly relative efficiency of $R_{\phi}(t)$ and $\bar{F}_{\phi}(t)$ is defined by $\frac{\operatorname{Var}\left(R_{\phi}(t)\right)}{\operatorname{Var}\left(\bar{F}_{\phi}(t)\right)}$. However, Figure 4 is based on RMSEs since Bayes estimators are biased. From Figure 4 (top left and right), it is evident that Bayes estimator $\bar{F}_{\phi}(t)$ is more efficient than the nonparametric estimator $R_{\phi}(t)$ both in the case of correctly specified prior measures and misspecified prior measures with smaller values of precision parameters.

\section{Illustrative Example}

We illustrate the proposed estimators with a randomly generated (synthetic) data set and compare it with nonparametric (PL-type) estimators. We consider thirty $(n=30)$ five-component parallel systems for the data generation purpose. Assume that $T_{i j} \sim \operatorname{Weibull}\left(\theta_{j}, \gamma_{j}\right)$, with

$$
\theta=(0.55,0.23,0.11,0.30,0.32) \text { and } \gamma=(1.08,0.80,1.79,0.82,1.57)
$$

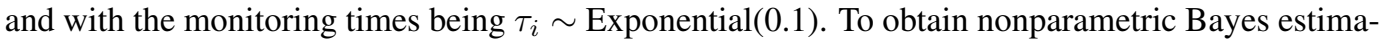
tor of system readability via components reliabilities we assumed PBDM priors where $\alpha_{j}(t, \infty)=$ $\beta_{j} \exp \left[-\left(\theta_{j} t\right)^{\gamma_{j}}\right]$ with $\theta=(1,1,1,1,1), \gamma=(1,1,1,1,1)$ and $\beta=(1,1,1,1,1)$ (Figure 5, right), that is, prior measures are misspecified. One can choose any other values of the parameters $\theta$ and $\gamma$ with the associated value of the precision parameter $\beta$ based on prior knowledge. In the absence of knowledge of the suitable PBDM prior measures, we may empirically estimate their parameters using the observed data. Equating the marginal reliability function of $T, \operatorname{Pr}(T>t)=\exp \left\{-(t \theta)^{\gamma}\right\}$ with the 75th and 50th percentiles of the reliability functions $R_{j, P L E}(t), j=1,2, \ldots, 6$, we obtain $\hat{\theta}=(0.59,0.19,0.10,0.71,0.40)$ and $\hat{\gamma}=(0.82,0.80,1.60,1.25,2.16)$. Again, following equation (12) of Rahman et al. (2014), we estimate $\beta$ by $\hat{\beta}=\sum_{i=1}^{M} D_{i} / \log (M) \approx(8,7,6,8,7)$, where $\sum_{i=1}^{M} D_{i}$ is the number of distinct uncensored observations for each component. The empirically estimated PBDM prior measure of system reliability function based on system data has $\alpha(s, \infty)=\beta \exp \left\{-(s \theta)^{\gamma}\right\}$, with $\hat{\beta}=6, \hat{\theta}=1.76$, and $\hat{\gamma}=0.08$. The resulting reliability function estimate with the empirically estimated prior measures with parameters $\hat{\theta}, \hat{\gamma}$ and $\hat{\beta}$ is presented in Figure 5(left panel). Figure 5]demonstrates that nonparametric Bayes estimates of system reliability function, in particular $\hat{\bar{F}}_{\phi}(t)$, performs better than other estimators being closer to the true reliability function. The right panel in Figure 5 indicates that the Bayes estimators are robust in the sense that the effect of prior misspecification is not severe when the precision parameters $\beta_{j}$ are small. 

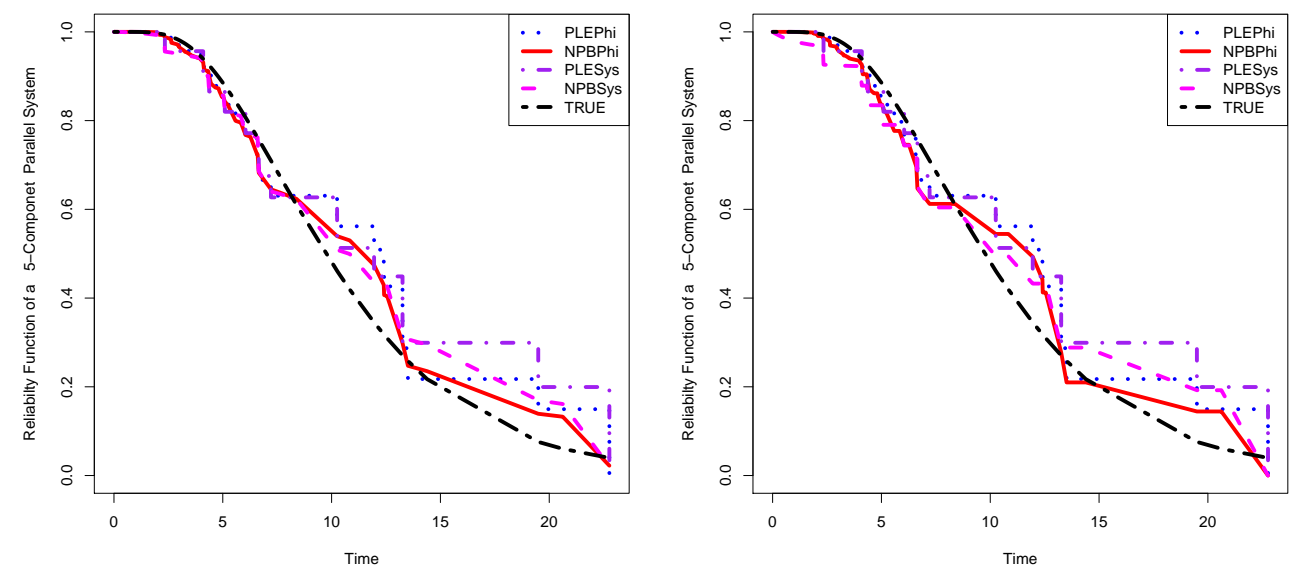

Figure 5: Reliability function of $\widehat{\bar{F}}_{N P B}(t)$ (labeled NPBSys), $\widehat{\bar{F}}_{\phi}(t)$ (labeled NPBPhi), $\widehat{R}_{P L E}(t)$ (labeled PLESys), and $\widehat{R}_{\phi}(t)$ (labeled PLEPhi) and the true distribution (labeled True). Priors are $\alpha(t, \infty)=$ $\beta \exp \left\{-(t \theta)^{\gamma}\right\}$ with $\theta=(0.59,0.19,0.10,0.71,0.40,0.08), \gamma=(0.82,0.80,1.60,1.25,2.16,1.76), \beta=$ $(8,7,6,8,7)$ (left side graph), $\theta=(1,1,1,1,1), \gamma=(1,1,1,1,1), \beta=(1,1,1,1,1)$ (right side graph).

\section{Concluding Remarks}

The estimator $\widehat{\bar{F}}_{\phi}(t)$ we obtained serves as the Bayesian counterpart of the Doss et al. (1989) estimator $\widehat{R}_{\phi}(t)$. We demonstrated that the Doss et al. (1989) estimator is a limiting case of our proposed estimator $\widehat{\bar{F}}_{\phi}(t)$. The Bayes estimators of the system reliability function are smoother in some sense than the corresponding nonparametric estimators. Simulation studies showed that $\widehat{\bar{F}}_{\phi}(t)$ yielded smaller RMSEs than $\widehat{R}_{\phi}(t)$. Simulation studies further demonstrated that, in terms of RMSEs, (see Figure 2 and Figure $33 \widehat{\bar{F}}_{\phi}(t)$ and $\widehat{R}_{\phi}(t)$ perform better than $\bar{F}_{N P B}(t)$ and $\widehat{R}_{P L E}(t)$, respectively. Nonparametric Bayes estimators are also more robust in the sense that the effect of prior misspecification is not severe when the precision parameters are small.

The class of PBDM prior measures is an elegant nonparametric prior which provides clear-cut posterior calculation even in the presence of incomplete observations. Under this PBDM priors, given the observations, which could be left-truncated, interval-censored, and right-censored, the posterior measure is also a PBDM. We derived closed-form Bayes estimators as well as developed a procedure to sample from the posterior measure. Moreover, PBDM priors could conveniently handle left-truncated, interval-censored, and right-censored data. However, we did not consider here the interesting and perhaps more realistic case where the component data are left truncated or interval-censored. In a forthcoming manuscript we will consider estimating the system reliability function under these more complicated data structures. 


\section{Acknowledgments}

Research is partially supported by NSF Grant DMS1106435 and NIH Grants R01 CA154731 and P20 RR17698. The authors are indebted to Professor James Lynch and Professor Jayaram Sethuraman for helpful discussions.

\section{References}

Barlow, R. E. (1984), "Reliability calculation and inductive inference: an overview," in Reliability theory and models (Charlotte, N.C., 1983), Orlando, FL: Academic Press, vol. 10 of Notes Rep. Comput. Sci. Appl. Math., pp. 221-229.

— (1985), "Combining component and system information in system reliability calculation," in Probabilistic methods in the mechanics of solids and structures (Stockholm, 1984), Berlin: Springer, pp. 375-383.

- (1986), "Mathematical theory of reliability. Historical perspectives," in Theory of reliability (Varenna, 1984), Amsterdam: North-Holland, Proc. Internat. School of Phys. Enrico Fermi, XCIV, pp. 3-11.

Barlow, R. E. and Hunter, L. C. (1960), “System efficiency and reliability,” Technometrics, 2, 43-53.

Barlow, R. E. and Marshall, A. W. (1967), "Bounds on interval probabilities for restricted families of distribution," in Proc. Fifth Berkeley Sympos. Mathematical Statistics and Probability (Berkeley, Calif., 1965/66), Vol. III: Physical Sciences, Berkeley, Calif.: Univ. California Press, pp. 229257.

Barlow, R. E. and Proschan, F. (1969), "A note on tests for monotone failure rate based on incomplete data." Ann. Math. Statist., 40, 595-600.

- (1981), Statistical theory of reliability and life testing: Probability Models, Silver Spring, MD: To Begin With.

- (1986), "Distributions with monotone failure rate," in Theory of reliability (Varenna, 1984), Amsterdam: North-Holland, Proc. Internat. School of Phys. Enrico Fermi, XCIV, pp. 12-22.

Boyles, R. A., Marshall, A. W., and Proschan, F. (1985), "Inconsistency of the maximum likelihood estimator of a distribution having increasing failure rate average," Ann. Statist., 13, 413-417.

Connor, R. J. and Mosimann, J. E. (1969), "Concepts of independence for proportions with a generalization of the Dirichlet distribution,” J. Amer. Statist. Assoc., 64, 194-206.

Doss, H., Freitag, S., and Proschan, F. (1989), "Estimating jointly system and component reliabilities using a mutual censorship approach,” Ann. Statist., 17, 764-782. 
El-Neweihi, E., Proschan, F., and Sethuraman, J. (1978), "Multistate coherent systems," J. Appl. Probab., 15, 675-688.

Esary, J. D., Marshall, A. W., and Proschan, F. (1970), "Some reliability applications of the hazard transform," SIAM J. Appl. Math., 18, 849-860.

- (1971), "Determining an approximate constant failure rate for a system whose components have constant failure rates," in Operations research and reliability (Proc. NATO Conf., Turin, 1969), New York: Gordon and Breach, pp. 195-210, with discussion by H. Reiche, M. Marcovici, Esary, Marshall and Proschan.

Esary, J. D. and Proschan, F. (1963), "Coherent structures of non-identical components," Technometrics, 5, 191-209.

Ferguson, T. S. (1973), "A Bayesian analysis of some nonparametric problems," Ann. Statist., 1, 209-230.

Grego, J., Li, S., Lynch, J., and Sethuraman, J. (2013), "Partition Based Priors and Multiple Event Censoring: An Analysis of Rosen's Fibrous Composite Experiment," Technometrics.

Hollander, M. and Peña, E. A. (1995), "Dynamic reliability models with conditional proportional hazards," Lifetime Data Anal., 1, 377-401.

— (1996a), "Dynamic reliability models," in Lifetime data: models in reliability and survival analysis (Cambridge, MA, 1994), Dordrecht: Kluwer Acad. Publ., pp. 131-140.

- (1996b), "Reliability models and inference for systems operating in different environments," Naval Res. Logist., 43, 1079-1108.

— (2004), "Nonparametric methods in reliability," Statist. Sci., 19, 644-651.

Hollander, M. and Proschan, F. (1984), "Nonparametric concepts and methods in reliability," in Nonparametric methods, Amsterdam: North-Holland, vol. 4 of Handbook of Statist., pp. 613655.

Kaplan, E. L. and Meier, P. (1958), "Nonparametric estimation from incomplete observations," $J$. Amer. Statist. Assoc., 53, 457-481.

Kvam, P. H. and Peña, E. A. (2005), "Estimating load-sharing properties in a dynamic reliability system," J. Amer. Statist. Assoc., 100, 262-272.

Kvam, P. H. and Samaniego, F. J. (1994), "Nonparametric maximum likelihood estimation based on ranked set samples,” J. Amer. Statist. Assoc., 89, 526-537.

Langberg, N., Proschan, F., and Quinzi, A. J. (1981), "Estimating dependent life lengths, with applications to the theory of competing risks," Ann. Statist., 9, 157-167. 
Meilijson, I. (1981), "Estimation of the lifetime distribution of the parts from the autopsy statistics of the machine," J. Appl. Probab., 18, 829-838.

Moeschberger, M. L. and David, H. (1971), "Life tests under competing cause of failure and the theory of competing risk," Biometrics, 27, 909-944.

Navarro, J. and Rychlik, T. (2007), "Reliability and expectation bounds for coherent systems with exchangeable components," J. Multivariate Anal., 98, 102-113.

Peña, E. A. and Hollander, M. (2004), "Models for recurrent events in reliability and survival analysis," in Mathematical reliability: an expository perspective, Boston, MA: Kluwer Acad. Publ., vol. 67 of Internat. Ser. Oper. Res. Management Sci., pp. 105-123.

Polpo, A. and Pereira, C. A. B. (2009), "Reliability nonparametric Bayesian estimation in parallel systems," IEEE Trans. Rel., 58, 364-373.

Polpo, A., Sinha, D., and Pereira, C. A. B. (2013), "Nonparametric Bayesian Estimation of Reliabilities in a Class of Coherent Systems," IEEE Trans. Rel., 62, 455-465.

Rahman, A. F., Lynch, J. D., and Peña, E. A. (2014), "Nonparametric Bayes estimation of gap-time distribution with recurrent event data," Journal of Nonparametric Statistics, 26, 575-598.

Sethuraman, J. (1994), “A constructive definition of Dirichlet priors," Statist. Sinica, 4, 639-650.

Sethuraman, J. and Hollander, M. (2009), "Nonparametric Bayes estimation in repair models," $J$. Statist. Plann. Inference, 139, 1722-1733.

Stokes, S. L. and Sager, T. W. (1988), "Characterization of a ranked-set sample with application to estimating distribution functions," J. Amer. Statist. Assoc., 83, 374-381.

Susarla, V. and Van Ryzin, J. (1976), "Nonparametric Bayesian estimation of survival curves from incomplete observations," J. Amer. Statist. Assoc., 71, 897-902.

Walker, S. and Muliere, P. (1997), "Beta-Stacy processes and a generalization of the Pólya-urn scheme," Ann. Statist., 25, 1762-1780.

\section{A Proofs}

Proof of Theorem 3: To see an explicit posterior measure we assume $H(\mathscr{B}, h, \mathscr{G})=\mathscr{D}(\mathscr{B}, h, \alpha)$, partition-based Dirichlet measure. Recall that right-censored sample $\left\{\left(v_{i}, \delta_{i}\right): i=1,2, \ldots, n\right\}$, where $\delta_{i}=1(0)$ means uncensored (right-censored) observation. That is $v_{i}=T_{i}$ when $\delta_{i}=1$ (uncensored observation) and $v_{i}>T_{i}$ when $\delta_{i}=0$ (right-censored). Without loss of generality let $T_{1}, T_{2} \ldots, T_{n-m+1}, T_{(1)}^{*}, T_{(2)}^{*}, \ldots, T_{(m-1)}^{*}$ be a sample from $P$ with $T_{1}, T_{2}, \ldots, T_{n-m+1}$ being the uncensored observations and $T_{(1)}^{*}, T_{(2)}^{*}, \ldots, T_{(m-1)}^{*}$ being the $m-1$ right-censored observations 
(ordered from smallest to largest). Consider $T_{(1)}^{*}, T_{(2)}^{*}, \ldots, T_{(m-1)}^{*}$ be the partition boundaries such that

$$
\mathscr{B}=\left(B_{1}, B_{2}, \ldots, B_{m}\right)
$$

is a measurable partition of $(0, \infty)$, where $B_{l}=\left(T_{(l-1)}^{*}, T_{(l)}^{*}\right], l=1,2,3, \ldots, m-1$, and $B_{m}=$ $\left(T_{(m-1)}^{*}, T_{(m)}^{*}\right)$ with $T_{(0)}^{*}=0$ and $T_{(m)}^{*}=\infty$. We consider $\mathscr{D}(\mathscr{B}, h, \alpha)$ with $h$ being of specific form (Dirichlet density function)

$$
h(\mathbf{y}) \propto\left[\prod_{l=1}^{m} y^{\alpha_{l}-1}\right] I\left\{\mathbf{y} \in R^{m}\right\},
$$

with $\alpha=\left(\alpha_{1}, \alpha_{2}, \ldots, \alpha_{m}\right), \alpha_{l}=\alpha\left(B_{l}\right), l=1,2, \ldots, m$.

By repeated application of Theorem 1 we obtain posterior distribution of $P$ given all complete (uncensored) observations $\left(T_{1}, T_{2} \ldots, T_{n-m+1}\right)$ which is also a PBDM $\mathscr{D}\left(\mathscr{B}, h^{\dagger}, \alpha^{*}\right)$, where

$h^{\dagger}(\mathbf{y}) \propto h(\mathbf{y})\left[\prod_{l=1}^{m} y_{l}^{\sum_{i=1}^{n-m+1} I\left(T_{i} \in B_{l}\right)}\right] \propto\left[\prod_{l=1}^{m} y^{\alpha_{l}-1}\right]\left[\prod_{l=1}^{m} y_{l}^{\sum_{i=1}^{n-m+1} I\left(T_{i} \in B_{l}\right)}\right] \propto\left[\prod_{l=1}^{m} y_{l}^{\alpha_{l}^{*}-1}\right]$, and $\alpha^{*}=\left(\alpha_{1}^{*}, \alpha_{2}^{*}, \ldots, \alpha_{m}^{*}\right)$ with $\alpha_{l}^{*}=\alpha_{l}+\sum_{i=1}^{n-m+1} I\left(T_{i} \in B_{l}\right)$.

Now suppose that $T_{n-m+2}$ is right-censored at $T_{(1)}^{*}$ (that is $\left.T_{n-m+2}>T_{(1)}^{*}\right)$ then it follows from the Theorem 2 that posterior distribution $\left(P \mid T_{(1)}^{*}\right)$ is $\operatorname{PBDM}, \mathscr{D}\left(\mathscr{B}, h^{\ddagger}, \alpha^{*}\right)$, where

$$
h^{\ddagger}(\mathbf{y}) \propto h^{\dagger}(\mathbf{y})\left(y_{2}+y_{3}+\ldots+y_{m}\right) \propto\left[\prod_{l=1}^{m} y_{l}^{\alpha_{l}^{*}-1}\right]\left[\sum_{j=2}^{m} y_{j}\right],
$$

and $\alpha_{l}^{*}=\alpha_{l}+\sum_{i=1}^{n-m+1} I\left(T_{i} \in B_{l}\right)$.

By repeated application of Theorem 2 for right-censored observations we get posterior distribution of $P$ given $\left.T_{(1)}^{*}, T_{(2)}^{*}, \ldots, T_{(m)}^{*}\right)$ is $\operatorname{PBDM}, \mathscr{D}\left(\mathscr{B}, h^{*}, \alpha^{*}\right)$, where

$$
h^{*}(\mathbf{y}) \propto h^{\dagger}(\mathbf{y}) \prod_{l=1}^{m}\left[\sum_{j=l+1}^{m} y_{j}\right] \propto\left[\prod_{l=1}^{m} y_{l}^{\alpha_{l}^{*}-1}\right] \prod_{l=1}^{m}\left[\sum_{j=l+1}^{m} y_{j}\right]
$$

and $\alpha_{l}^{*}=\alpha_{l}+\sum_{i=1}^{n-m+1} I\left(T_{i} \in B_{l}\right)$.

Thus the posterior measure, $P \mid\left(T_{1}, T_{2} \ldots, T_{n-m}, T_{(1)}^{*}, T_{(2)}^{*}, \ldots, T_{(m)}^{*}\right)$ is the PBDM, $\mathscr{D}\left(\mathscr{B}, h^{*}, \alpha^{*}\right)$, where

$$
h^{*}(\mathbf{y}) \propto h(\mathbf{y})\left[\prod_{l=1}^{m} y_{l}^{\sum_{i=1}^{n-m+1} I\left(T_{i} \in B_{l}\right)}\right] \prod_{l=1}^{m}\left[\sum_{j=l+1}^{m} y_{j}\right] \propto\left[\prod_{l=1}^{m} y_{l}^{\alpha_{l}^{*}-1}\right] \prod_{l=1}^{m}\left[\sum_{j=l+1}^{m} y_{j}\right],
$$

and $\alpha_{l}^{*}=\alpha_{l}+\sum_{i=1}^{n-m+1} I\left(T_{i} \in B_{l}\right)$.

Received: July 12, 2020

Accepted: November 20, 2020 